\title{
Effective mechanical and transport properties of polysiloxane matrix based composites
}

\author{
M. Maršálková1, S. Urbanová ${ }^{2}$ J. Salačová ${ }^{1} \&$ M. Šejnoha ${ }^{2}$ \\ ${ }^{I}$ Faculty of Textile Materials, Technical University in Liberec, \\ Czech Republic \\ ${ }^{2}$ Department of Mechanics, Faculty of Civil Engineering, \\ Czech Technical University in Prague, Czech Republic
}

\begin{abstract}
The present paper describes experimental and numerical work for the evaluation of the selected effective mechanical and transport material properties of polysiloxane matrix based composites. Three different material systems including carbon, basalt and glass plain weave textile reinforcements are first studied experimentally with emphasis on the influence of processing temperature, number of reinforcement layers and the resulting porosity. The Mori-Tanaka averaging scheme is then selected for the numerical evaluation of effective electric conductivities adopting the standard bottom-up hierarchical homogenization.

Keywords: homogenization, ceramic textile composites, Charpy test, falling test, electric conductivity, porosity, Mori-Tanaka method.
\end{abstract}

\section{Introduction}

Textiles are no longer a sole realm of clothing industry but diffuse in many other lines of business and branches of science. Nowadays, an increasing number of fiber reinforced composite components are being fabricated with load-carrying fibers, which are woven to form a fabric. This reinforcement system has advantages with respect to fabrication as well as mechanical properties.

In this regard, carbon-carbon $(\mathrm{C} / \mathrm{C})$ textile composites have enjoyed a considerable interest as potential candidates for high-tech applications including rocket industry on the one hand and bone-surgery on the other hand. However, their expansion into points of practical applications has been obstructed by a number of 
unfavorable properties typical of $\mathrm{C} / \mathrm{C}$ composites, both mechanical and biological, such as relatively high brittleness or their susceptibility to release carbon particles when exposed to living organism causing inflammation of human tissue.

Recently, a new class of materials has emerged showing similar applicability as $\mathrm{C} / \mathrm{C}$ composites. These material systems derived from polysiloxane matrices belong to an important class of ceramic matrix based composites. In view of biomechanical applications, a number of studies have already suggested acceptable mechanical properties of these "inexpensive" material systems comparable to human bones together with their satisfactory biocompatibility and bioactivity [1]. Their potential use in high temperature applications has been discussed in [2].

Regardless of their final use, most of these systems share a common denominator being the dependence of their properties on the processing rout. This objective is addressed hereinafter in its preliminary form. The results of the initiated experimental program are presented first. In this framework, the impact damage resistance is examined in the light of the type of reinforcement, the number of reinforcement layers and processing temperature. To that end, the standard Charpy and falling weight [3] tests were performed considering six and eight layer polysiloxane matrix based composite reinforced by carbon, basalt and glass plain weave fabrics and manufactured at three different processing temperatures $\left(200^{\circ}, 600^{\circ}, 1100^{\circ} \mathrm{C}\right)$. Numerical simulation of these tests is still at its infancy and is typically reduced to the analysis of macroscopically homogeneous orthotropic systems [4] irrespective of the actual microstructure.

This is partly attributed to the geometrical complexity of the reinforcements, which is often of random nature and its proper introduction into advanced computations calls for the definition of a certain statistically equivalent periodic unit cell (SEPUC) [5]. This is not a trivial task and requires extensive image analysis to acquire basic geometrical data and consequently well defined experimentally measurable data to corroborate the numerical predictions. The latter issue considers effective electric conductivities already exploited to some extent in the combined numerical-experimental study of unidirectional basalt [3] and carbon-textile [6] polysiloxane matrix based composites. Detailed analysis of SEPUC goes, however, beyond the present scope. The former modeling issue will, therefore, be investigated in a simplified manner in conjunction with the Mori-Tanaka mean-field theory successfully applied in a number of our previous works $[7,8$, to cite a few].

\section{Experimental program}

The experimental program considers three types of composites with carbon, basalt and glass plain weave textile reinforcements manufactured by molding together six and eight layers of fabric first impregnated by the silicon resin Lukosil M130 (polymethylsilicone resin dissolved in xylene) and cured at $200^{\circ} \mathrm{C}$ for $6 \mathrm{~h}$ to form the polymer precursors. To investigate the influence of temperature these specimens having in-plane dimensions $12 \times 12 \mathrm{~cm}$ were further treated at $600^{\circ} \mathrm{C}$ and $1100^{\circ} \mathrm{C}$ in vacuum. Unfortunately, the curing step at $1100^{\circ} \mathrm{C}$ produced 
specimens which were too brittle, essentially falling in pieces thus ruling out any mechanical testing. The results, presented in the remaining part of this section, are therefore only preliminary more suggesting further directions of this ongoing research than allowing us to draw any solid conclusions.

The project objectives include quantification of these systems from the mechanical response as well as transport properties point of view. While the presented mechanical tests aim at addressing the impact damage tolerance, the transport parameters consider effective electric conductivities only to provide macroscopic data for validation of the proposed homogenization scheme.

\subsection{Charpy test}

The Charpy impact test serves to evaluate the energy absorption capability of the material. It consists of a pendulum with a defined mass, which upon swinging hits the specimen at its middle span length while transferring the kinetic energy to it. In this particular case a CEAST Resil Impactor device was used resulting in an impact speed of about $3.3 \mathrm{~m} / \mathrm{s}$ of a $15 \mathrm{~J}$ hammer. The $12 \times 12 \mathrm{~cm}$ laminated plate was cut into $(l \times h) 40 \times 10 \mathrm{~mm}$ unnotched specimens in both warp and weft directions. Six specimens were tested for each direction and laminate thickness (6 and 8 layers). The energy $E_{c}$ absorbed by the specimen was recorded and the Charpy impact strength $\alpha_{c}$ was calculated as [3]

$$
\alpha_{c}=\frac{E_{c}}{l h}
$$

where $l$ and $h$ are the specimen length and height,respectively.The results are stored in Table 1. Similar results for carbon textile reinforcements bonded to an epoxy resin were reported in [4] showing an increasing trend in impact resistance with increasing specimen thickness. Note that for short and long basalt fiber reinforced thermoplastic matrix laminates the Charpy impact strength was found in the range of $48-75 \mathrm{~kJ} / \mathrm{m}^{2}$ [3] comparable to the values obtained for glass fabric based systems.

Table 1: Results of the Charpy test for specimens cured at $200^{\circ} \mathrm{C}$ - Absorbed energy $[\mathrm{J}] /$ Impact strength $\left[\mathrm{kJ} / \mathrm{m}^{2}\right]$.

\begin{tabular}{|l|c|c|c|c|}
\hline \multirow{2}{*}{ Reinforcement } & \multicolumn{2}{|c|}{ warp (No. of layers) } & \multicolumn{2}{c|}{ weft (No. of layers) } \\
\cline { 2 - 5 } & 6 & 8 & 6 & 8 \\
\hline Carbon & $5.4 / 135$ & $5.7 / 143$ & $5.4 / 135$ & $7.4 / 186$ \\
Basalt & $6.2 / 156$ & $9.8 / 244$ & $6.9 / 173$ & $5.5 / 138$ \\
Glass & $2.1 / 51$ & $3.8 / 95$ & $2.9 / 72$ & $3.0 / 74$ \\
\hline
\end{tabular}




\subsection{Falling test}

The falling weight test serves to evaluate the material ductility or toughness. In the present study, specimens having in-plane dimensions $60 \times 60 \mathrm{~mm}$ were subjected to impact of a falling weight, see [3] for instrumentation details. The material is typically quantified by calculating the penetration energy written as

$$
E_{P}=\frac{E_{\max }}{h},
$$

where $E_{\max }$ is the energy absorbed by the specimen and $h$ is the material thickness. This quantity allows us to compare the results received for various laminates with a variable thickness. The measure of ductility is provided by the ductility index given by

$$
D_{i}=\frac{E_{\max }-E_{F \max }}{E_{\max }} \times 100 \%,
$$

where $E_{F \max }$ is the energy associated with the maximum value of the force reached during experiment. The high brittleness of the material is indicated by the value of $D_{i}$ approaching 1 . On the contrary, materials with high toughness have the values of $D_{i}$ close to 0 .

The corresponding values of the two parameters are stored in Table 2. Inspecting the values for penetration energy may indicate a certain increase in brittleness of the matrix phase with increasing temperature. This, however, is contradicted by the calculated values of $D_{i}$. Such a discrepancy can be attributed to an insufficient number of samples cured at $600^{\circ}$ and their partial damage even prior to testing. Nevertheless, we may still conclude that material properties of the matrix phase do not change much in this particular range of temperature. This is also supported by the measurements of effective electric conductivities suggesting the transition of polymeric matrix into a ceramic at much higher temperatures well exceeding $1000^{\circ} \mathrm{C}$.

Table 2: Results of the Weight falling test: Penetration energy $[\mathrm{kJ} / \mathrm{m}]$ / Ductility Index [\%].

\begin{tabular}{|l|c|c|c|c|}
\hline \multirow{3}{*}{ Reinforcement } & \multicolumn{4}{|c|}{ Temperature $\left[{ }^{\circ} \mathrm{C}\right]$} \\
\cline { 2 - 5 } & 200 (No. of layers) & 600 (No. of layers) \\
\cline { 2 - 5 } & 6 & 8 & 6 & 8 \\
\hline Carbon & $49 / 58$ & $45 / 59$ & $39 / 59$ & $58 / 37$ \\
Basalt & $56 / 59$ & $53 / 55$ & $30 / 56$ & $30 / 39$ \\
Glass & $47 / 56$ & $39 / 50$ & $29 / 50$ & $43 / 65$ \\
\hline
\end{tabular}




\subsection{Electric conductivities}

The HP 4339B High Resistance Meter designed for measuring very high resistance and related parameters of insulation materials was utilized to determine the through thickness resistivities of individual laminates. Knowing the electrode area $S_{e l}$ and the thickness of each specimen $h$ the resulting resistivities follow from

$$
\rho^{\mathrm{mes}}=\frac{R^{\mathrm{mes}} S_{e l}}{h},
$$

where $R^{\text {mes }}$ is the measured resistance. The averages calculated from ten measurements taken for each specimens are stored in Table 3. It is interesting to point out that in the through-thickness direction all specimens show height resistivity regardless of the type of reinforcement (note the through thickness measurements of individual textile fabrics available in the last column of the table for illustration). Thus the flow in this direction is dominated by the matrix phase which is highly resistive for polymers as oppose to pyrolyzed ceramics. This is further evident when comparing the results for specimens cured at $600^{\circ}$ and $1100^{\circ} \mathrm{C}$. Since the matrix resistivities were not directly measured, the presented results can be exploited together with the homogenization analysis, see Section 3, to estimate this property in the sense of an inverse approach.

Table 3: Through thickness effective electric resistivities $\left[\Omega \mathrm{cm} \times 10^{9}\right]$.

\begin{tabular}{|l|c|c|c|c|c|c|c|}
\hline \multirow{3}{*}{ Reinforcement } & \multicolumn{6}{|c|}{ Temperature $\left[{ }^{\circ} \mathrm{C}\right] /$ No. of layers } \\
\cline { 2 - 8 } & \multicolumn{2}{|c|}{200} & \multicolumn{2}{|c|}{600} & 1100 & fabric \\
\cline { 2 - 7 } & 6 & 8 & 6 & 8 & 6 & 8 & - \\
\hline Carbon & 435 & 332 & 311 & 362 & 9 & 5 & $1.12 \times 10^{5}$ \\
Basalt & 471 & 356 & 347 & 399 & 9 & 7 & $2.61 \times 10^{12}$ \\
Glass & 550 & 408 & 458 & 340 & 9 & 9 & $2.15 \times 10^{12}$ \\
\hline
\end{tabular}

\subsection{Image analysis}

The last part of the experimental program was concerned with the measurements of basic geometrical parameters of a single ply composite marked in Figure 2(a) for an idealized periodic unit cell (PUC). It will be seen in the next section that these parameters can be linked to an optimal equivalent ellipsoidal inclusion needed in the Mori-Tanaka averaging scheme.

The image analysis device used in the present study for structural image acquisition and analysis consisted of NIKON ECLIPSE E 600 microscope, Märzhauser motorized scanning stage, digital monochrome camera VDC 1300C 


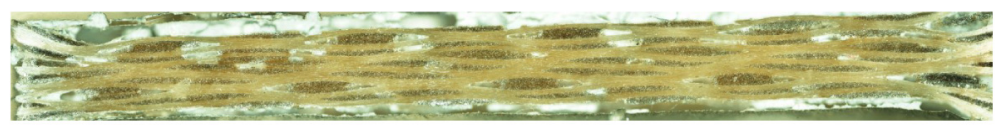

Figure 1: Example of a compound image - glass fabric based composite.

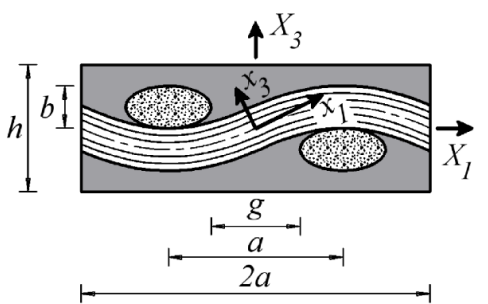

(a)

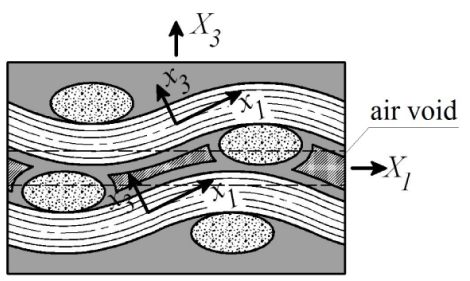

(b)

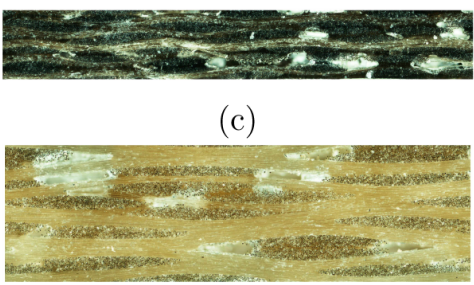

(d)

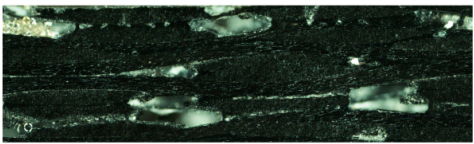

(e)

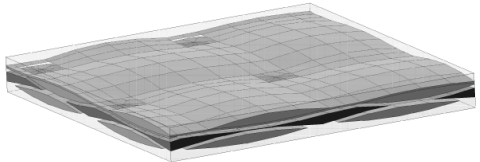

(f)

Figure 2: (a) Single ply PUC, (b) Two layer SEPUC; Examples of six layer composites: (c) basalt, (d) glass, (e) carbon reinforcements; (f) Computational model of SEPUC.

and image analysis software LUCIA G (www.lim.cz). The final image was obtained by compounding several images in both horizontal and vertical directions. Although assuming identical properties of plain weave fabrics in both directions, such images were created independently for the warp as well as weft directions to eliminate small geometrical deviations associated with the manufacturing of fabrics. One particular example is presented in Figure 1. Images of all three composite systems are available in Figs. 2(c)-(e) for comparison. Notice also a relatively large amount of intrinsic porosity for all specimens. Particular values are stored in Table 4.

The basic parameters of the periodic unit cell were found manually as averages of $50 \div 100$ measurements. The resulting statistics combining both 6 and 8 layer systems as well as warp and weft directions are listed in Table 4.

Exploitation of these parameters is twofold. First, they can be utilized in a certain optimization step to derive a two-layer SEPUC schematically displayed in Figure 2(b), which apart from other geometrical imperfections accounts also 
Table 4: Parameters of the periodic unit cell.

\begin{tabular}{|l|c|c|c|c|c|c|c|c|c|}
\hline \multirow{3}{*}{ Reinforcement } & \multicolumn{7}{|c|}{ Parameter $[\mu \mathrm{m}]$} & \multirow{2}{*}{$\begin{array}{c}\text { Porosity } \\
{[\%]}\end{array}$} \\
\cline { 2 - 10 } & \multicolumn{2}{|c|}{$\mathrm{a}$} & \multicolumn{2}{|c|}{$\mathrm{b}$} & \multicolumn{2}{|c|}{$\mathrm{g}$} & \multicolumn{2}{c|}{$\mathrm{h}$} & \\
\cline { 2 - 10 } & $\mu$ & $\sigma$ & $\mu$ & $\sigma$ & $\mu$ & $\sigma$ & $\mu$ & $\sigma$ & \\
\hline Carbon & 2123 & 90 & 157 & 9 & 387 & 39 & 307 & 24 & 9.9 \\
Basalt & 1075 & 92 & 107 & 11 & 175 & 46 & 223 & 25 & 11.4 \\
Glass & 2080 & 106 & 119 & 11 & 600 & 108 & 278 & 22 & 9.6 \\
\hline
\end{tabular}

for mutual shift and nesting of individual layers clearly visible in Figs. 2(c)(e). This approach is described in detail in [5] including the introduction of the porous phase to create the actual computational model presented in Figure 2(f). This approach, embracing also X-ray microtomography and numerical analysis based on Extended Finite Element method, is a subject of an ongoing research and will not be pursued therein. Instead, the Mori-Tanaka method will be adopted to predict the effective properties. In such a case, the four parameters are used to quantify the geometry of an equivalent ellipsoidal inclusion. An extensive study carried out in $[7,8]$ suggested that the values of $\xi_{2}, \xi_{3}$ axes, given $\xi_{1}=1$ (note that the Eshelby solution of a single inclusion problem depends only on the mutual ratio of the ellipsoid semi-axes), can be related to $g / a$ and $b / a$ ratios as

$$
\xi_{2} \approx \frac{1}{7}\left(1-\frac{g}{a}\right), \quad \xi_{3} \approx \frac{1}{60}\left(1-4 \frac{b}{a}\right) .
$$

\section{Numerical evaluation of effective electric conductivities}

This section outlines derivation of the effective electric conductivities in the light of uncoupled hierarchical modeling. In particular, a two step homogenization is performed independently on the scale of fiber tow and the scale of a single ply composite. In both cases, a two-phase composite is considered such that a matrixinclusion system free of porous phase is evaluated first. Porosity is introduced next in the second homogenization step into a new homogenized matrix. While on the level of yarns the fibers and pores are represented by infinite cylinders with circular and elliptical cross-sections, respectively, the ply level assumes an equivalent ellipsoid described by Eq. (5) to represent the fiber tow and oblate spheroids to account for large inter-tow vacuoles seen in Figs. 2(c)-(e). Note that the effective conductivities of the fiber tow needed on the ply scale are not measured, but predicted from homogenization. Classical lamination theory, simple rule of mixtures, is finally adopted to arrive at effective properties of the layered composite.

Owing to space limitation, we outline the essential theoretical background of the Mori-Tanaka method for the level of fiber tow only to review the basic terminology 
and refer the interested reader to [8] for the more complex case of an orthotropic textile ply.

\subsection{Mori-Tanaka method}

Referring to the underlying problem of transport of electric charge and following [9] the method builds upon the solution of an isolated inclusion (generally assumed elliptical) in an unbounded matrix subjected to an average electric field intensity $\left\langle\boldsymbol{e}_{1}\right\rangle=-\left\langle\nabla \varphi_{1}\right\rangle$ ( $\varphi$ being the electric potential) found in the matrix.

As mentioned above it is sufficient to consider a two-phase composite with subscript 1 reserved for the matrix and 2 for the inclusion. The local electric field intensity in the inclusion $\left\langle\boldsymbol{e}_{2}\right\rangle$ (uniform for elliptical shape) can be then written in terms of the partial concentration factor $\mathbf{A}_{2}^{\text {dil }}$ as

$$
\left\langle\boldsymbol{e}_{2}\right\rangle=\mathbf{A}_{2}^{\text {dil }}\left\langle\boldsymbol{e}_{1}\right\rangle
$$

Standard averaging yields

$$
\langle\boldsymbol{e}\rangle=\sum_{r=1}^{2} c_{r}\left\langle\boldsymbol{e}_{r}\right\rangle \quad \text { and } \quad\langle\boldsymbol{j}\rangle=\sum_{r=1}^{2} c_{r}\left\langle\boldsymbol{j}_{r}\right\rangle
$$

where $j$ is the current density vector and $c_{r}$ stands for the volume fraction of a given phase. The local and macroscopic constitutive equations written in terms of local $\sigma_{r}$ and homogenized $\sigma^{\text {hom }}$ electric conductivities follow from Ohm's law in the form

$$
\left\langle\boldsymbol{j}_{r}\right\rangle=\boldsymbol{\sigma}_{r}\left\langle\boldsymbol{e}_{r}\right\rangle \quad \text { and } \quad\langle\boldsymbol{j}\rangle=\boldsymbol{\sigma}^{\text {hom }}\langle\boldsymbol{e}\rangle .
$$

With reference to [8] we write

$$
\mathbf{A}_{2}^{\text {dil }}=\left[\mathbf{I}-\mathbf{S}\left(\boldsymbol{\sigma}_{1}\right)^{-1}\left(\boldsymbol{\sigma}_{1}-\boldsymbol{\sigma}_{2}\right)\right]^{-1},
$$

where $\mathbf{S}$ is the depolarization tensor of cylindrical inclusion and $\mathbf{I}$ represents the identity matrix. Next, introduce the localization factor $\mathbf{A}_{2}^{\mathrm{MT}}$ such that

$$
\left\langle\boldsymbol{e}_{2}\right\rangle=\mathbf{A}_{2}^{\mathrm{MT}}\langle\boldsymbol{e}\rangle
$$

which in combination with Eqs. (7) 1 and (6) reads

$$
\mathbf{A}_{2}^{\mathrm{MT}}=\mathbf{A}_{2}^{\mathrm{dil}}\left[c_{1} \mathbf{I}+c_{2} \mathbf{A}_{2}^{\mathrm{dil}}\right]^{-1}
$$

The effective electric conductivity then directly follows from Eq. (7) $)_{2}$ in the form

$$
\boldsymbol{\sigma}^{\mathrm{hom}}=\boldsymbol{\sigma}^{\mathrm{MT}}=\boldsymbol{\sigma}_{1}+c_{2}\left(\boldsymbol{\sigma}_{2}-\boldsymbol{\sigma}_{1}\right) \mathbf{A}_{2}^{\mathrm{MT}} .
$$




\subsection{Results}

Consider Table 3 to recall high resistivity of basalt and glass fibers. We thus considered only carbon reinforcements for numerical analysis. The local properties of individual phases stored in Table 5 were taken from literature, see [6, and references therein].

Table 5: Material parameters of individual phases.

\begin{tabular}{|c|c|}
\hline Material & Electric conductivities [S/m] \\
\hline Carbon fibers & $\left(5.9 \cdot 10^{4}, 5.9 \cdot 10^{2}, 5.9 \cdot 10^{2}\right)$ \\
Polysiloxane matrix at $200^{\circ} \mathrm{C}$ & $8 \times 10^{-11}$ \\
Voids filled with air & $10^{-10}$ \\
\hline
\end{tabular}

This computational part was carried out with the help of $\mathbf{C E}_{\mathrm{L}} \mathbf{P}$ program developed at the Department of Mechanics of the Faculty of Civil Engineering, the Czech Technical University in Prague (http://ksm.fsv.cvut.cz/web/). The results for the system cured at $200^{\circ} \mathrm{C}$ are given in Table 6 .

Table 6: Effective electric conductivities [S/m].

\begin{tabular}{|c|c|c|c|}
\hline \multirow{2}{*}{$\begin{array}{c}\text { material } \\
\text { vol. frac. } \rightarrow\end{array}$} & \multicolumn{2}{|c|}{ Equivalent inclusion } & \multirow{2}{*}{ Electric conductivities } \\
\cline { 2 - 3 } & fiber|tow & void & $\sigma_{X_{1}}, \sigma_{X_{2}}, \sigma_{X_{3}}$ \\
\hline Fiber-matrix & $\infty, 1,1$ & - & $4.0 \times 10^{4}, 4.2 \times 10^{-10}, 4.2 \times 10^{-10}$ \\
Porous yarn & - & $\infty, 1.6,10$ & $3.7 \times 10^{4}, 3.7 \times 10^{-10}, 4.0 \times 10^{-10}$ \\
\hline Tow-matrix & $0.1,0.01,1$ & - & $1.8 \times 10^{4}, 1.8 \times 10^{4}, 2.6 \times 10^{-10}$ \\
Porous ply & - & $0.33,1,1$ & $1.5 \times 10^{4}, 1.5 \times 10^{4}, 3.4 \times 10^{-10}$ \\
\hline Laminate (MT) & - & - & $1.2 \times 10^{4}, 1.2 \times 10^{4}, 1.9 \times 10^{-10}$ \\
Laminate (EXP) & - & - & $\sigma_{X_{3}}=2.8 \times 10^{-10}$ \\
\hline
\end{tabular}

It is seen that the results provided by homogenization are in a relatively good agreement with laboratory measurements, compare with Table 3 after setting $\sigma_{x_{3}}^{\text {mes }}=1 / \rho^{\text {mes }}$. Such encouraging results motivated us finally to the prediction of electric conductivity of the pyrolyzed matrix combining homogenization and experimental measurements to get $\sigma_{m}^{1100^{\circ} \mathrm{C}} \approx 9 \times 10^{-9}[\mathrm{~S} / \mathrm{m}]$.

\section{Conclusion}

The present paper is concerned with the experimental and computational investigation of polysiloxane matrix based textile composites. The first part 
of experimental program showed a relatively good impact damage resistance particularly at the low curing temperature of $200^{\circ} \mathrm{C}$. To confirm such observations for moderate and high curing temperatures will require, however, additional testing. The influence of polymer-ceramic transition was further examined from the electric conductivity point of view confirming a considerable increase of electric conductivity for pyrolyzed ceramics.

Numerical modeling aimed at supporting the ability of hierarchical homogenization to predict sufficiently accurately the effective properties of plain weave textile composites. For simplicity, we limited our attention to the MoriTanaka averaging scheme. A reasonably good agreement between experimental and numerical results is encouraging particularly in view of more complex computational approach based on the application of statistically equivalent periodic unit cells. This subject is under current investigation.

\section{Acknowledgement}

The financial support provided by the GAČR grant No. 105/11/0224 is gratefully acknowledged.

\section{References}

[1] Suchý, T., Balík, K., Černý, M., Sochor, M., Hulejová, H., Pešáková, V. and Fenclová, T., A composite based on glass fibers and siloxane matrix as a bone replacement. Ceramics-Silikáty, 52(1), pp. 29-36, 2008.

[2] Černý, M., Glogar, P., Nekoksa, P. and Sucharda, Z., Thermally resistant composites with R-glass fibres and polysiloxane-derived ceramic matrix. Bulletin of the Czech Society for Carbon Materials, 1, 2004.

[3] Deák, T., Czigány, T., Maršálková, M. and Militký, J., Manufacturing and testing of long basalt fiber reinforced thermoplastic matrix composites. Polymer Engineering and Science, 50(12), pp. 2448-2456, 2010.

[4] Hufenbach, W., Marques Ibraim, F., Langkamp, A., Böhm, R. and Hornig, A., Charpy impact tests on composite structures - an experimental and numerical investigation. Composites Science and Technology, 68, pp. 2391-2400, 2008.

[5] Vorel, J., Zeman, J. and Šejnoha, M., Homogenization of plain weave composites with imperfect microstructure: Part II-analysis of real-world materials. International Journal for Multiscale Computational Engineering, 2012. Submitted, available at http://arxiv.org/abs/1001.4063.

[6] Vorel, J., Maršálková, M., Urbanová, S. and Šejnoha, M., Experimental and numerical derivation of effective electric conductivities for polysiloxanematrix based composites. CC2011, Chania, Crete, Greece 6-9 September 2011, ed. B. Topping, Civil-Comp Press, 2011.

[7] Skoček, J., Zeman, J. and Šejnoha, M., Effective properties of CarbonCarbon textile composites: application of the Mori-Tanaka method. Modelling and Simulation in Materials Science and Engineering, 16(8), p. paper No. $085002,2008$. 
[8] Vorel, J. and Šejnoha, M., Evaluation of homogenized thermal conductivities of imperfect carbon-carbon textile composites using the Mori-Tanaka method. Structural Engineering and Mechanics, 33(4), pp. 429-446, 2009.

[9] Benveniste, Y., A new approach to the application of Mori-Tanaka theory in composite materials. Mechanics of Materials, 6, pp. 147-157, 1987. 